\title{
TSOU: A combined Traffic Scheduling, Optimization and Utility function based on Sailfish optimized DCNN in a Smart Grid (SG) communication network
}

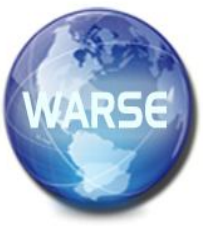

\author{
Sabita Nayak ${ }^{1 *}$, Amit Kumar ${ }^{2}$ \\ ${ }^{* 1}$ Assistant Professor, Department of Electronics and Communication, \\ B.I.T. Sindri, University-JUT, Dhanbad, Jharkhand, India \\ *Email: sabitanayak0305@gmail.com \\ ${ }^{2}$ Assistant Professor, Department of Electrical Engineering, \\ B.I.T. Sindri, Dhanbad, University-JUT, Ranchi, Jharkhand India
}

\begin{abstract}
The rapid advances in networking technologies aspect foremost crisis in terms of the system reliability, efficiency, sustainability and cost in concert with the wireless sensor communication network. This communication platform integrated with Smart Grid system to transverse us the information about the grid system failure, fault detection, retrieval, security, monitoring and management of the energy bases with assurance. At present, cognitive radio networks (CR) is vital among the wireless technology for a smart grid communication system with less hindrance and proficient bandwidth.This paper aims at introducing a contemporary data traffic scheduling framework in a CR-SG communication network based on an advanced sailfish optimized Deep Convolutional neural network (SF-DCNN) for an effectual Smart grid communication system. Here, perhaps a strengthened DCNN is unwrapped to schedule the traffic data and so elements using the selfish optimizer algorithm to achieve less latency with maximum throughput. The results of the analysis demonstrate that the proposed approach on the SG system will executes substantially better scheduling than all other perceived models, suggesting that the prototype proposed is well suited to standardized the communication in a SG system with less interference.
\end{abstract}

Key words: Traffic scheduling, Smart grid (SG), Cognitive radio network (CRN), Sail Fish Optimizer (SFO), DCNN

\section{INTRODUCTION}

In this communication era, smart grid is one among the perceptive control system over sensors. The conventional power grid system is a centralized, interconnected massive network with lot of control system, which delivers the consumer a seamless unidirectional power supply of electricity [1]. But still this unidirectional power system lacks in various aspects such as to monitor, control, effective generation and reliability to come up with an intelligent functional component for smart working of the power grid system [2]. Instance the supervisory control and data acquisition (SCADA) technology is utilized by the conventional power grid system to have a centralized operation, processing and storage with a challenge of performance degradation in capability of controlling the power distribution network in real time [3].Hence as a development smart grid 2 and 3 are evolved but these systems will drastically increase the demand of power and un reliability. In order to overcome these challenges effective system architecture with intellectual algorithms and Mechanism are required [4].

The intelligent monitoring of the power equipment, fault diagnosing and management of the smart power grid system can be attained over and done with wireless sensor network (WSN) podium [5, 6]. In addition, application of wireless multimedia sensor networks (WMSN) and Cognitive radio (CR) technology in smart grid system partakes much consideration these days due to its intelligent ability to bestow intense stakeout information and upgrading band usage [7, 8]. The pros of these vital technologies throw together an astonishing place in architecture of a SG system which is further in need of huge bandwidth and network resources [9, 10]. The need to meet the heterogeneous communication while unexpected environment conditions with high reliability and low latency is the light for the success of CR based smart grid systems [11]. But on the other side, large amount of data transferring with high quality of Services (QoS) through the CR based communication network is in great demand of bandwidth and energy resources with an astonishing interference over limited radio frequency $[12,13]$. For example, if there is a smart camera around the solar power plant to monitor the intensity and directions of the panels, then the authority will manage the solar panel directions from the control room itself based on provided video surveillance by the camera. So that an ultra-smart grid system with multimedia transmission without any interference is essential to operate, control and monitor the SG system resourcefully [14]. Hence, we need a way out to these catastrophes in SG 
applications impels the research intellectuals towards this vicinity.

In this paper, a scheduling methodology based on a deep convolutional neural network algorithm for the prediction of data traffic in the SG system is proposed. Here we mainly focus on the latency and throughput values of the smart grid applications and it was spotlighted into several applications with diverse network bandwidths. Then based on the priority queues an effectual SG data traffic scheduling is carried out based on Deep Convolutional Neural Network (DCNN) [15] whose parameters are optimized based on a Sailfish Optimizer [16] to minimize and maximizes the latency and throughput correspondingly. The leftover of this article is coordinated as follows: Section 2 defines the SG based communication network techniques that provide various works pertaining to the use of upgrading techniques and technologies. Section 3 defines the proposed methodology, demonstrates the traffic scheduling outline based on deep convolutional neural network and Sailfish optimizer. Section 4bestows the simulation results. Section 5expresses the conclusion.

\section{RELATED WORK: A BRIEF REVIEW}

Over the past few years, quite a lot of traffic scheduling schemes using different methods and strategies has been developed in SG communication technology for power distribution networks without any loss and interference. Among them some of the works of research scholars were reviewed here in this section.

Markkula (2020) et al. in [17], has researched about the demand response traffic and its possibility through the SG communication network. As a conclusion from his obtained results, based on some applied limitations in the traffic volume and the radio channel, the public LTE network is much suitable for this perception. They simulated the proposed approach using Riverbed Modeler Network by using the propagation models, suburban topology and LTE functionality. Their simulation outcome is satisfactory with 99\% of packet delivery ratio on behalf of SG DR (UL) traffic and SG DR control (DL) traffic was about $100 \%$ even at the time of peak traffic. At the time of analysing case 2 and 3, occurred a maximum delay of about $331 \mathrm{~s}$ and a considerable interference. And they suggest enhancing the performance of LoRaWAN through optimization.

Radzi (2019) et al. in [18] has suggested an CATScha algorithm for scheduling the heterogeneous traffic demands in a power distribution network based on its varying conditions. This can be achieved through Qos, Prioritizing and optimizing the left behind resources. They implement the suggested approach by using the NS-3 simulator and it was validated by using the MATLAB and analysed by varying the traffic priority. The results demonstrate us that the throughput and link efficiency can be achieved with less delay, when comparing our approach with the conventional traffic scheduling approaches with no Context aware. They suggested optimizing the utilization and consumption of energy in future research works.

Akerele (2019) et al. in [19] has integrated the WSN and ONU for implementing the adaptive scheduling mechanism for high priority traffic with more reliability and less end to end delay. Through simulation, QoS mechanism implemented has shown less $40 \%$ of packet delay, more reliability and lower end to end delay in an Fi-WSN system and in the Long-Reach Passive Optical Networks (LR-PONs). In addition, they have achieved the $33 \%$ delay reduction at full load condition in EPON and a delay occurred was below the level of tolerance in the SG atmosphere. They suggest a multi-hop WSN interface with fibre network and an optimization model to enhance the reliability of the data packets with higher priorities for further future research.

Yadav (2017) et al. in [20] has studied about the CR based heterogeneous traffic in SG communication network. Then based on the QoS, SG data traffic was sub classified into diverse priority classes. Blocking, dropping and interference probability, and call completion rate are utilized to analyse their suggested scheme. Pareto distribution model is utilized and analysed based on the shape parameter by them instead of Poisson process model in order to estimate the traffic at heavy tailed traffic. The performance of the suggested scheme was analysed with and without priority settings of traffic data and it is observed that the analysis based on priority outperforms better than the system without priority settings in the SG communication network.

Jiang (2017) et al. in [21] has developed a traffic model of Smart Grid Electronic Data and multimedia video over Cognitive Radio technology. Here, in order to lessen the communication burden, they follow the triggered Gaussian distribution to classify the KF data with high priority and a buffer mechanism to improve the delivery probability of data by resending them along with the new arrival. To overcome the buffer overflow problem, the un-transferred data were compressed; reduced and new data are assuaged to neglect the state of congestion in the CR network. A New Binary Exponential Back off (NBEB) algorithm was utilized by them to compress the unsent, temporal selection of new data and to lessen the arrival traffic rate with less attempt of failure. Finally, their proposed NDEB result analysis literally lowers the probability of blocking and upsurges the probability of success in order to enhance the communication performance. Yigit (2016) et al. in [22], has proposed a LQ-CMST and PCA-MC, a routing and scheduling algorithm for SG applications. The performance analysis of the suggested approach was evaluated under the harsh smart grid channel conditions based on the modulation and encoding schemes. Finally, it was concluded that the comparative analysis consequently lessens the delay at various channel and network traffic load conditions than that of the conventional approaches. And they suggest to improve the fairness by 
combining their suggest approach with fair scheduling strategies as a future research work.

Yu (2015) et al. in [23] has studied about the QoS scheduler problem which was responsible for maintaining the resources and transmission in the CR-based SG communication network. They prioritize the user demand by its role and situation of the SG to provide every one the QoS with less transmission delay. So that they make use of semi-Markov decision process by adaptive heuristic dynamic programming methodology was utilized for the entire transmission scheduling problem. At last their simulation outcome ensured the differential QoS with less transmission delay in the SG communication network.

Garlapati (2015) et al. in [24] has concentrated on the AMI network data and suggested a soft MAC protocol for scheduling by employing SDMAC and OTRA-THS MAC meant for random data collection. Markov chain was suggested to analyse the proposed protocol and the simulation results were analysed based on the metrics like average throughput and packet delay. Hence from the analysis, it was concluded that they achieve a less control overhead of about $2144 \%$, latency of power consumption data about $1 / 3^{\text {rd }}$ and latency of power outage data collection about $1 / 8^{\text {th }}$ when comparing it with the 3G CDMA2000. As a result, they increased the smart meters in the base station with less requirement of bandwidth and has the capability to lessen the CAPEX and OPEX of a utility owned AMI network

\section{PROPOSED METHODOLOGY}

\subsection{CR-SG Communication network}

In the era of communication, Smart grid (SG) network shown in figure 1 is one of the emerging networks under consideration for various applications, in which the WS nodes in the whole region have to be covered by its infrastructure to have interference less communication. Thus, the entire SG network is further sub-classified as follows [25]:

- Home Area Network (HAN)

- Neighbourhood Area Network (NAN)

- Wide Area Network (WAN)

The enclosure of cognitive radio (CR) in the above mention sub networks will ensure the efficient diverse communication by allocating the successful spectrums.

The HAN network which acts as bottom layer of the communication network will interrogate between the home appliances to have an effective consumption of energy. The HAN network (e.g.; smart meter) fortified with CR turns out into HAN cognitive gateway (HGW) which contributes for level of reliable communication with less interference. The NAN network mostly inducted in substations (PMUs and DGM) mainly focus on the distribution sectors. To transverse huge amount of data to the control centres such as distributed energy resources along with Phasor measurement unit data it acts as middle layer and bridge between the WAN and HAN cognitive gateways. The upper most layer of the SG communication network is WAN which mainly focus on generation and transmission sectors (renewable) to gathers the info of several NAN gateways to transfer it to control centres for long distance communication. Apart from these CR gateways there are numerous components in the SG communication network integrated with $\mathrm{CR}$ system architecture. By this integration the Spectral Efficiency (SE) of the system will gets improved by lowering the SNR when compared it with the regular system without CR [26].

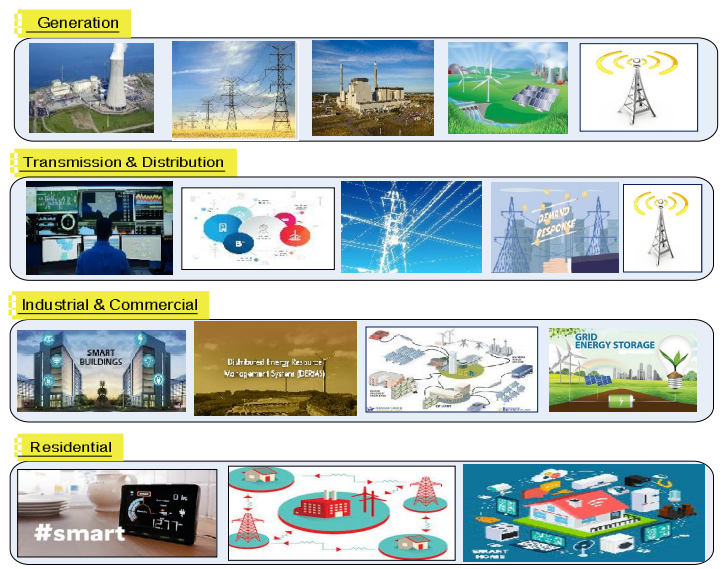

Figure 1: CR-SG communication Network

An extensive assortment of communication appliances makes use of these SR technologies, such as Advanced Metering Infrastructure (AMI), Demand Response Management (DRM), Phasor Measurement Units (PMUs), Distributed Grid Management (DGM), Supervisory Control and Data Acquisition (SCADA) systems and Electric Vehicles (EV) [27]. At this juncture, the AMI is a two-way communication system with notable load management; DRM makes use of old assets to handle demand, generation and equalization of loads. In case of any overloaded condition in the grid system, distribution mechanism scheme will restrict and manages the load demand of the consumer by means of smart meter and appliances. Phasor Measurement Units (PMUs) are used by Wide-Area Situational Awareness (WASA) for recording any incidents or as to collect data efficiently. The Distributed Grid Management (DGM) system make use of SCADA to automatically detects the crises in multiple regions and also used to control and monitor the grid systems. The Electric Vehicles (EV) will act as a backup storage to help us out to meet the energy demand. Mostly the CR-SG communication network reliant on sensing spectrums, allocation and managements at the end of Cognitive radio gateways (CRG). In this paper, we consider that the sensing of spectrum is carried out at the base station (BS) and the Smart grid Communication Node (SCN) will offer the info of available channel specifics. In the direction of encounter the QoS requirement of the entire SG applications, efficient system reliability, throughput and latency is necessary and this challengeable task is under our consideration while 
scheduling the traffic data in the CR-SG communication network in this paper.

\subsection{Suggested Traffic Scheduling Scheme}

In this paper, we intend to propose a Sailfish optimized DCNN based scaffold intended for data traffic scheduling in Cognitive Radio (CR)-based SG communication network. Our proposed traffic scheduling process comprises of subsequent phases: (I) Classification of SG Data (II) Scheduling Process (II) Combined Utility Function.

1) Classification: In this section, we present the classification of SG data based on the QoS requirement i.e. Latency and Throughput. In general, to meet the reliability requirement of the system, we need to maximize the throughput and minimize the latency value at similar time. Here, we classify the $\mathrm{SG}$ communication nodes ( $\mathrm{SCN}$ ) into two classes based on the throughput and latency values. Let $L_{a}, L_{b}, L_{c}$ be the classes of Latency requirement group and $D_{a}, D_{b}$ be the classes of throughput requirement group. Table 1 depicts the range requirement and corresponding SG applications of latency and throughput. Moreover, besides upon the nature, SCN's data is further classified based on the priority (higher to lower level) is depicted clearly in Table 2 .

Table 1: Classes based on the requirement of Latency and Throughput

\begin{tabular}{|c|c|c|c|}
\hline & Classes & Description & Applications \\
\hline \multirow[b]{3}{*}{ 导 } & $L_{a}$ & $\begin{array}{l}\text { High priority } \\
L \leq 100 \mathrm{~ms}\end{array}$ & DGM, WASA \\
\hline & $L_{b}$ & Medium priority & DRM, DER \\
\hline & $L_{c}$ & $\begin{array}{l}\text { Low Priority } \\
L>1 s \\
H P>L P, 100 m s<\end{array}$ & \begin{tabular}{|l} 
AMI, EV \\
\end{tabular} \\
\hline \multirow{2}{*}{ 音 } & $D_{a}$ & $\begin{array}{l}\text { High priority } \\
500<D<1500 k b p\end{array}$ & $\begin{array}{l}\text { WASA, AMI } \\
\text { [NAN/WAN], DER, } \\
\text { DRM [NAN/WAN] }\end{array}$ \\
\hline & $D_{b}$ & $\begin{array}{l}\text { Low priority } \\
D<500 \mathrm{kbps}\end{array}$ & $\begin{array}{l}\text { AMI [HAN], DER, } \\
\text { EV, DGM }\end{array}$ \\
\hline
\end{tabular}

Table 2: Classification based on priority

\begin{tabular}{|c|c|l|}
\hline S. No & Type of Data & \multicolumn{1}{c|}{ Description } \\
\hline $\mathbf{1}$ & IE & $\begin{array}{l}\text { Interrupt emergency } \\
\text { (interrupt by PU) }\end{array}$ \\
\hline $\mathbf{2}$ & E & Emergency \\
\hline $\mathbf{3}$ & I & $\begin{array}{l}\text { Interrupt (Non emergent SCN } \\
\text { data) }\end{array}$ \\
\hline $\mathbf{4}$ & R & $\begin{array}{l}\text { Recurrent (retransmission of } \\
\text { SCN data) }\end{array}$ \\
\hline $\mathbf{5}$ & NA & Newly Arrived \\
\hline
\end{tabular}

On the basis of the above priority classification mentioned in table 2 they are further sub classified as similar to earlier latency and throughput requirements sub classes. But the difference here is to classify based on the available channel bandwidth (i.e. from low to high) and it is represented as CBJ , If $n=0 \rightarrow$ Low Bandwidth and for $n=1 \rightarrow$ Low Bandwidth , where, $\mathrm{J}=0,1,2,3 \ldots \ldots . .7$.In case, if there is an availability of low channel bandwidth then in converse for low throughput application high level priority is given to evade transmission delay and damage caused while transmitting crucial data. Similarly, in case, if there is an availability of high channel bandwidth then in converse for high throughput application, low level priority is given. So that we can met the latency requirement of the data transmitted by utilizing available high bandwidth channel. Outline of our proposed scheme based on priority classes of bandwidth is shown in Figure 2

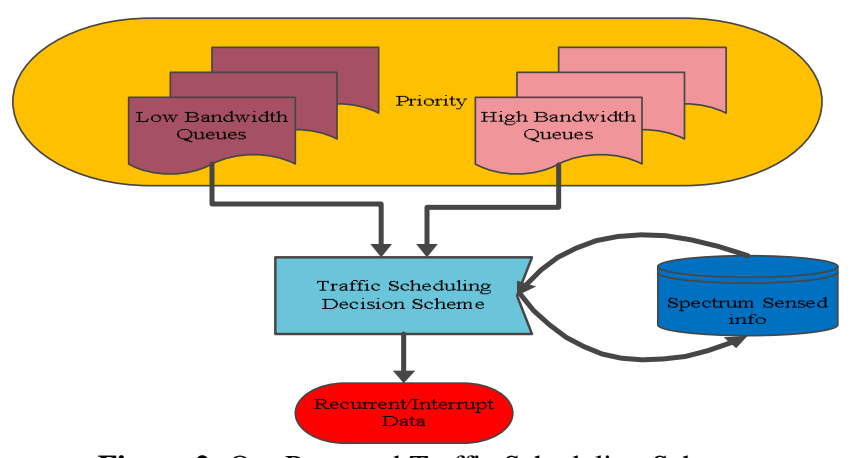

Figure 2: Our Proposed Traffic Scheduling Scheme

2) Scheduling process: In this subdivision, we describe about the traffic scheduling (allocation of channel) and formation of queue scheme based on its priority by arranging the SCN data by considering its nature $(\mathrm{E} / \mathrm{I} / \ldots / \mathrm{R})$ in order to meet the $\mathrm{QoS}$ requirement. Here, the SCNs will be in queuewaiting for the slot (fixed time interval) to which is allocated for the particular SCN and the packets to be transmitted throughthis slot varies based on the channel bandwidth. To avoid the transmission interruption of SCN the primary user (PU) will attain at the start of time slot. The 'channel occupancy vector' is represented as $\mathrm{CV}(\mathrm{n})$, where, $\mathrm{n}=0$ or 1 , ' $^{\prime}$ ' denotes the occupancy of channel by PU and ' 1 ' denotes the availability of channel with no PU. The component 'State" represents the current status of the SCN with number of packets to be transmitted, channel occupancy and associated flags (E, I...., $\mathrm{R})$. The channel state vector is represented as cSNR and the product of packet size (p) and probability of bit error $(\mathrm{pb})$ will gives us the probability of retransmission. The channel allocation for SCN in the queue was carried out by the scheduler by considering the allocation policy $\gamma(n)$. Here, no channel is allocated when $\gamma(n)=0$ and when $\gamma(n)=i$, then $\mathrm{i}^{\text {th }}$ channel is allocated to the $\mathrm{n}^{\text {th }} \mathrm{SCN}$. If there is an availability of non-empty packets in the SCNs, a decision space will be formulated based on decision policies. The overall working of the proposed traffic scheduling scheme is shown in figure 2 . An efficient operation of the traffic scheduling scheme is 
accomplished by satisfying the subsequent general weight equation of SCN is displayed as in equation (1).

$w_{n}=w_{i, n}^{k}+w_{R} 1_{\left(R_{n}=1\right)}+w_{l} 1_{\left(l_{n}=1\right)}+w_{R l} 1_{\left(R_{n}=1, l_{n}=1\right)}$

$+w_{E} 1_{\left(E_{n}=1\right)}+w_{R E} 1_{\left(R_{n}=1, E_{n}=1\right)}+w_{l E} 1_{\left(l_{n}=1, E_{n}=1\right)}$

$+w_{R I E} 1_{\left(R_{n}=1, l_{n}=1, E_{n}=1\right)}$

where $w_{i, n}^{k}$ denotes the new SCN in the queue and $l_{a-1}=\left\{\begin{array}{ll}1, & \text { if } a=1 \\ 0, & \text { otherwise }\end{array}\right\}$ is an indicator function.

During the arrival of the SCN, the allocation policy will be initialized so that the updating of state vector, allocation, interruption, recurrent data transmission where carried out simultaneously.

3) Combined utility Function: The utility function of our suggested traffic scheduling scheme is to satisfy the user with delay less QoS requirement by considering the latency and throughput to improve the fair scheduling among the consumers while formulating the utility function.

Latency: Delay caused during the scheduling process will be zero, when there is an availability of channel and it will be allocated to the particular SCN. Similarly, the delay time will be $\mathrm{T}$, when there is no allocation of channel. Consequently, the transmission delay of each and every SCN will be evaluated and if there is no availability channel to allocate then,

$T_{n}(m)= \begin{cases}\Delta T, & \text { no channel } \\ 0, & \text { else }\end{cases}$

The utility function of overall latency with respect to al the SCNs are evaluated by using the equation

$\left.\rho_{L}(s(m)), \Gamma(m)\right)=\left\{\begin{array}{l}\sum_{n=1}^{N} w_{n}(m) T_{n}(m), \text { if } \alpha_{n}(m)>0 \\ 0, \text { else }\end{array}\right.$

where

$$
\begin{aligned}
& \rho_{L} \text {-Utility Function of Latency } \\
& s(m) \text {-State Vector } \\
& \text { Г(n) -Decision space } \\
& \alpha_{n}(m+1)=\left\{\begin{array}{ccc}
\alpha_{n}(m)-p, & \text { if } & \gamma_{n}=1,2, \ldots L \\
\alpha_{n}(m)-3 p, & \text { if } & \gamma_{n}=L+1 \ldots . C
\end{array}\right.
\end{aligned}
$$

where $c=L+H$

Throughput: The throughput utility function can be represented as $\rho_{R}$ and for a fixed slot of time the throughput will be low or high based upon its bandwidth values. Hence, while allocating SCN if a channel is with low bandwidth means then its throughput value will gets improvedby $R_{1}$, in transverse for a channel with high bandwidth means then its throughput value will gets improved by $R_{2}$. For no allocation of channel means then the throughput value will be zero. The utility function of overall Throughput with respect to al the SCNs are evaluated by using the equation (5)

$\left.\rho_{R}(s(m)), \Gamma(m)\right)=\left\{\begin{array}{l}\sum_{n=1}^{N} w_{n}(m) R_{n}(m), \text { if } \quad \alpha_{n}(m)>0 \\ 0, \text { else }\end{array}\right.$

where

$\rho_{R}$

-Utility Function of Throughput

$s(m)$-State Vector

$\Gamma(m)$-Decision space

$R_{n}(m)= \begin{cases}R_{1}, & \text { if } \gamma_{n}=1,2, \ldots \ldots, L \\ R_{n 2}, & \text { if } \gamma_{n}=L+1,2, \ldots, C \\ 0 & \text { else }\end{cases}$

Here the main contribution of our proposed algorithm is to lessen and exploit the latency and throughput utility functions correspondingly. Our final combined utility function is the total sum of both the latency and throughput utility functions; hence our overall objective becomes the minimization problem. Therefore, the combined utility function equation can be represented as follows:

$\rho(m)=\beta_{L} \rho_{L}(m)-\beta_{R} \rho_{R}(m)$

where $\beta_{L}$ and $\beta_{R}$ - Selected weights of $\rho_{L}$ and $\rho_{R}$ for prioritizing

\subsection{Optimization Strategy}

In this section, initially we originate the optimization problem for our proposed CR-SG-traffic scheduling process. Later, a Deep Convolutional neural network [21] is employed for the solution of this optimization problem. Here, Sail fish (SF) Optimizer is utilized for the generation of optimized weights for hidden and output layer of DCNN shown in figure 3 . Hence, our suggested strategy will optimize the weight parameters to demoralize the overall system cost.

$J=\sum_{i=0}^{\infty} \rho(s(i), \Gamma(i))$

The DCNN is low interpreted approach used to solve various engineering problems and its ability to learn and process the huge data is the major advantage of this technique. Here, privacy threat and random weight updating increases the difficulty. The input to our DCNN optimizer is the system state $S$ and the output will be the overall system cost in between the weight parameters were updated by means of SF. The various layers of DCNN are described as follows.

Input Layer: This layer holds the input of our approach as system state 's'.

Convolution Layer: In this layer based on the weights updated kernel filters were built and it is represented as 
$x_{0, f 1}^{l}=f\left[\sum_{i \in m} X_{i}^{l-} * K_{i o, f 1}^{l}+B^{1}\right]$

Where, $m=n-k+1$

\section{$f(x)$ - Activation Function}

Here we make use of activation function named rectified linear unit (ReLU) is employed among Tanh, Leaky RELU, etc. which is faster among all the three in deep learning.

$$
f_{\operatorname{Re} L U}(x)=\max (0, x)
$$

Pool Layer: In the DCNN, the man function of this pool layer is to diminish the dimensions of data, so as to improve the computation and reduces the storage memory and over fitting. Max pooling and Average pooling are the two common pooling layers, among that we make use of max pooling because of its performance achievements.

$$
x_{0,}^{l}=f\left[\max \left(\sum_{i \in m} X_{i}^{l-1}\right)+B^{1}\right]
$$

Batch Normalization (BN) layer: In this layer, we process and distribute evenly the input of each layer before training so that we will achieve a better and efficient training speed with less overfitting

$$
y_{1}=\gamma * \frac{x_{i}-\mu_{x}}{\sqrt{\sigma_{x}^{2}+\epsilon}}+\beta
$$

where $\mu_{x}$-Mean Value

$\sigma^{2} x$-Variance

$\gamma \beta$ - General terms

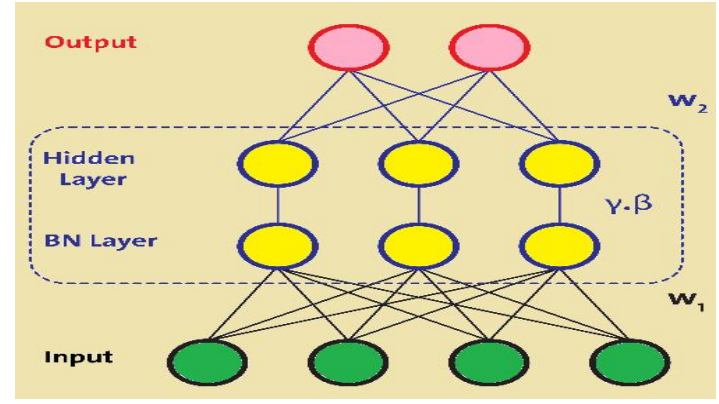

Figure 3: Structure of DCNN

Fully-Connected Layer: Dense and Soft max layer are the part of fully connected layer. In this layer the input of the previous layer is taken to compute the learnable parameter D and the equation is expressed as follows.

$$
x_{0}^{l}=f\left(x_{i}^{l-1} * D_{i o}^{l}+B^{l}\right.
$$

The softmax value is represented as follows,

$$
S_{i}=e^{Z i} /\left(\sum_{1}^{j} e^{z j}\right)
$$

Next, we propose a SF Optimizer to generate the optimal weight for the hidden and output layer of DCNN. The group hunting strategy of this optimizer helps to solve a wide range of unsolved problems in engineering field. In this SF algorithm [28], it is assumed that the SF are applicant solutions and the Weights are the position of sailfish in the search space. Initially, we create the weight solution space indiscriminately and the optimizer can search through the hyper dimensional spaces for mutable position vectors. Let the initial position of SF be $S F_{i, k} \in R(i=1,2, \ldots \ldots . . m)$. Here, the weight parameter of the DCNN is updated by using the following SF equation.

$W_{n}^{1}=W_{\text {elite }}^{i}-\lambda_{i} *\left(\operatorname{rand}(0,1) *\left(\frac{W_{\text {elite }}^{i}+W_{\text {inj }}^{i}}{2}\right)-W_{\text {old }}^{i}\right)$

where $W_{\text {elite }}^{i}$ - Best Position yet

$W_{i n j}^{i}$-Best target

$W_{\text {old }}^{i}$ - Current Position of SF

$\lambda$ - Coefficient

The coefficient parameter will be ' 1 , when the rand value $>0.5$, ' -1 ' when rand value is $<0.5$ and ' 0 ' when rand value is equal to 'zero'. These instabilities and updating of SF positions accentuate the global search solutions. By updating the weights of DCNN by SF optimizer the system cost of the proposed traffic scheduling framework can be optimized with the optimal decision policy.

\section{RESULT AND DISCUSSION}

The proposed data traffic scheduler for a Smart Grid communication network has been evaluated through extensive simulation. Simulation has been carried out under priority and non-priority conditions for some appliances to explore the latency and throughput. We have considered the CR-SG network with respect to the Primary User (PU)/ Channels, SCNs at a desired bandwidth condition. The arrival of PU will reside in the channel for 8 consecutive slots. The desired applications for analysis are WASA, DGM, DER and EV. The weights for optimizer network are optimized by SF optimizer and they are utilized for initialization and those output weight will be generated as the scalar value for each slot. These slots will be sub classified into two sets as training and testing sets for the DCNN. Hence the optimized neural network-based traffic scheduling in the SG communication network will shows an optimized system cost based on its corresponding learning rate (We assume that $\mathrm{LR}=0.01$ ). In Fig 4, it shows the convergence of cost functions for Learning Rate (LR) with respect to the time slots.

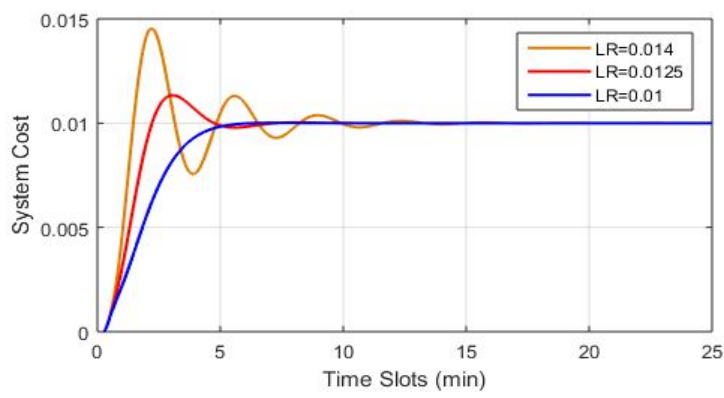

Figure 4: System cost analysis based on learning rate 
Figure 5 illustrates the analysis of four SCNs from varies class based on Latency and primary user arrival rate. If there is a less traffic of PU then the availability of channel to the SCNs will be more. Here our priority flows as follows: WASA, DRM, DGM and EV and it is observed that the SCN with highest priority has less latency and the SCN with low priority has high latency.

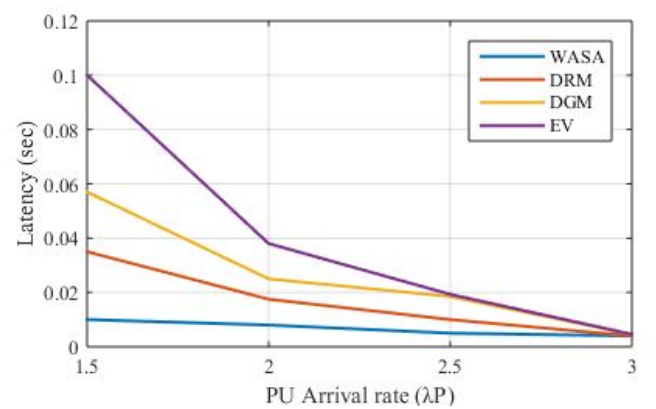

Figure 5: Analysis of Latency for some appliances

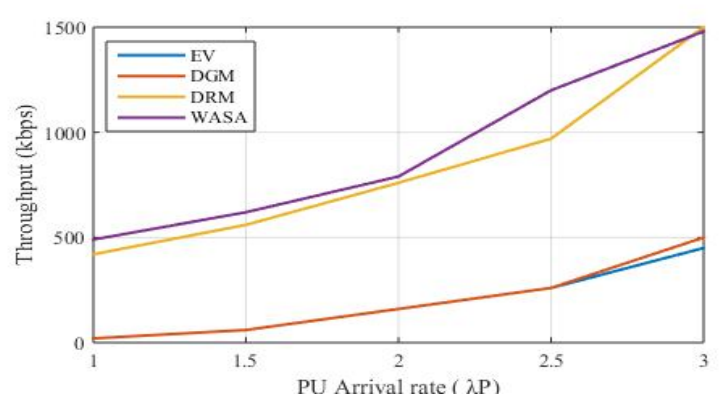

Figure 6: Analysis of Throughput for some appliances

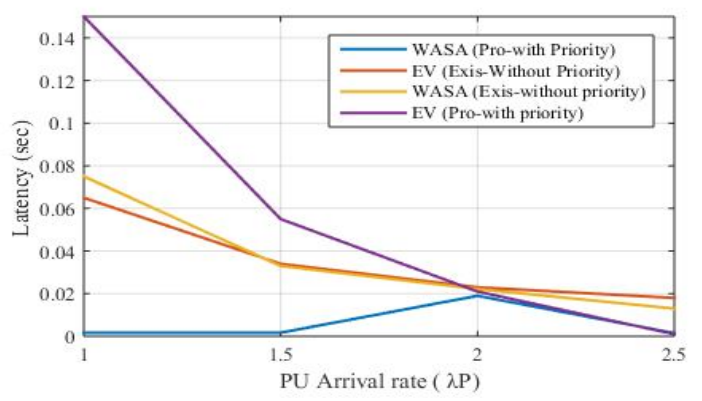

Figure 7: Analysis of Latency for proposed and existing approach with and without priority conditions

For all the four SCNs the latency is achieved beneath the desired value except DGM due to its higher bandwidth it achieves the looked-for latency at about $\lambda p \geq 1.75$. Here we can achieve the desired throughput with no conceding the latency obligation of the SCNs which can be seen in figure 6 plotted against the Primary user arrival rate. It is clearly depicted in the figure that there is an increase in throughput for lower PU traffic and observed that except for WASA remaining appliances like DRM, DGM and EV has the throughput value less than $500 \mathrm{kbps}$ when $\lambda \mathrm{p}=1$. ie. For
WASA -500kbps, for DRM -450kbps, and the throughput for $\mathrm{EV}$ and DGM is $100 \mathrm{kbps}$ respectively.

Figure 7 and 8 compares the latency and throughput statistics of proposed and existing scheduling approaches [29] in the CR-SG communication network. It can be seen that our proposed scheduler is performing well under the concept of cost, latency and throughput fairness provisioning. As a result, fairness while scheduling is well maintained, so that the proposed overall system achieves less latency and max throughput than existing CR-SG scheduling approach.

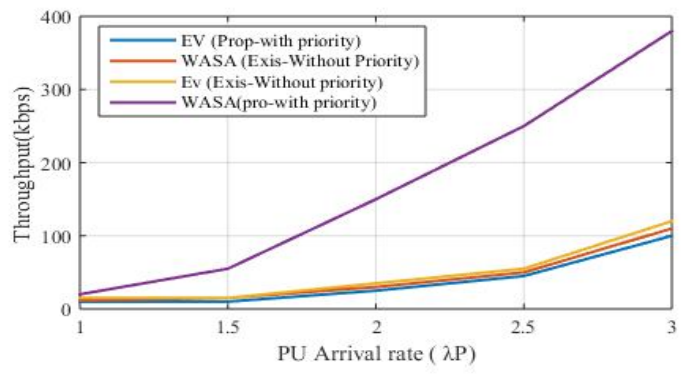

Figure 8: Analysis of Throughput forproposed and Existing approach with and without priority conditions

In figure 7 for SG application WASA and EV the latency plotting against primary user arrival rate is shown with and without priority conditions. Even at the worse traffic condition WASA has achieved the required latency and the latency will be same for both the application when no priority is assigned to the SCN. Similar results will be observed for throughput of the same applications. The efficiency of the proposed traffic scheduler can be analysed by means of throughput i.e by the system utilization of resources. By inspecting the latency and throughput curve, our proposed schedulers give the great performance even at the time of emergency and have been serviced with less loss.

\section{CONCLUSION}

In this paper, the data traffic scheduling framework based on priority in a CR based SG communication networkare portrayed in an efficient model that comprises the best attributes of DCNN and SF Optimizer to increase performance and preserve the unique traits of our proposed scheme. It achieves an optimal system cost and decision policy by minimizing the latency and maximizing throughput based on the combined utility function. Finally, from the simulation outcome shown, it is concluded that the proposed traffic scheduling scheme for CR-SG application is successful in meeting the QoS requirement.

\section{REFERENCES}

1. N. Kayastha, D. Niyato, E. Hossain and Z. Han. Smart grid sensor data collection, communication, and networking: a tutorial, Wireless Communications 
and Mobile Computing, vol. 14, no. 11, pp. 1055-1087, July 2012.

https://doi.org/10.1002/wcm.2258

2. S. Howell, Y. Rezgui, J. Hippolyte, B. Jayan and H. Li. Towards the next generation of smart grids: Semantic and holonic multi-agent management of distributed energy resources, Renewable and Sustainable Energy Reviews, vol. 77, pp. 193-214, Sep.2017.

3. Y. Huang, S. Werner, J. Huang, N. Kashyap and V. Gupta. State Estimation in Electric Power Grids: Meeting New Challenges Presented by the Requirements of the Future Grid, IEEE Signal Processing Magazine, vol. 29, no. 5, pp. 33-43, Aug. 2012.

https://doi.org/10.1109/MSP.2012.2187037

4. K. Moslehi and R. Kumar. Smart Grid - a reliability perspective, 2010 Innovative Smart Grid Technologies

5. Gungor, B. Lu and G. Hancke Opportunities and Challenges of Wireless Sensor Networks in Smart Grid, IEEE Transactions on Industrial Electronics, vol. 57, no. 10, pp. 3557-3564,Feb. 2010.

https://doi.org/10.1109/TIE.2009.2039455

6. E.Sivanantham, Base Station Positioning in Wireless Networks Using Self-Adaptive Particle Swarm Optimization Approach, International Journal of Advanced Trends in Computer Science and Engineering, vol. 9, no. 2, pp. 1662 - 1667, March-April 2020.

https://doi.org/10.30534/ijatcse/2020/116922020

7. I. Akyildiz, T. Melodia and K. Chowdhury. A survey on wireless multimedia sensor networks, Computer Networks, vol. 51, no. 4, pp. 921-960,March 2007.

8. A. Ali and W. Hamouda. Advances on Spectrum Sensing for Cognitive Radio Networks: Theory and Applications, IEEE Communications Surveys \& Tutorials, vol. 19, no. 2, pp. 1277-1304, Nov. 2017. https://doi.org/10.1109/COMST.2016.2631080

9. Honggang Wang, Yi Qian and H. Sharif. Multimedia communications over cognitive radio networks for smart grid applications, IEEE Wireless Communications, vol. 20, no. 4, pp. 125-132, Sep.2013.

10. H. Akshatha, H. V. Balachandra Achar, Dual Parametric Stabilization of Interference and Throughput in Wireless Sensor Network- Optical Communication, International Journal of Advanced Trends in Computer Science and Engineering, vol. 8, no. 5, pp. 1937-1945, September - October 2019 https://doi.org/10.30534/ijatcse/2019/18852019

11. V. Gungor et al., A Survey on Smart Grid Potential Applications and Communication Requirements, IEEE Transactions on Industrial Informatics, vol. 9, no. 1, pp. 28-42, Sep.2013.

12. M. Erol-Kantarci and H. Mouftah. Energy-Efficient Information and Communication Infrastructures in the Smart Grid: A Survey on Interactions and Open Issues, IEEE Communications
Surveys \& Tutorials, vol. 17, no. 1, pp. 179-197, July 2015.

https://doi.org/10.1109/COMST.2014.2341600

13. A. Khan, M. Rehmani and M. Reisslein. Cognitive

Radio for Smart Grids: Survey of Architectures, Spectrum Sensing Mechanisms, and Networking Protocols, IEEE Communications Surveys \& Tutorials, vol. 18, no. 1, pp. 860-898,Ep. 2016.

14. S. Alam, M. Sohail, S. Ghauri, I. Qureshi and N. Aqdas. Cognitive radio based Smart Grid Communication Network, Renewable and Sustainable Energy Reviews, vol. 72, pp. 535-548,Aug. 2017.

https://doi.org/10.1016/j.rser.2017.01.086

15. S. Shadravan, H. Naji and V. Bardsiri. The Sailfish Optimizer: A novel nature-inspired metaheuristic algorithm for solving constrained engineering optimization problems, Engineering Applications of Artificial Intelligence, vol. 80, pp. 20-34, 2019.

16. S. Wang and $\mathrm{H}$. Chen. A novel deep learning method for the classification of power quality disturbances using deep convolutional neural network, Applied Energy, vol. 235, pp. 1126-1140, Feb. 2019.

17. J. Markkula and J. Haapola. Shared LTE Network Performance on Smart Grid and Typical Traffic Schemes, IEEE Access, vol. 8, pp. 39793-39808, Feb. 2020.

18. N. Radzi et al., Context Aware Traffic Scheduling Algorithm for Power Distribution Smart Grid Network, IEEE Access, vol. 7, pp. 104072-104084, July 2019.

19. M. Akerele, I. Al-Anbagi and M. Erol-Kantarci. A Fiber-Wireless Sensor Networks QoS Mechanism for Smart Grid Applications, IEEE Access, vol. 7, pp. 37601-37610, Mer. 2019.

https://doi.org/10.1109/ACCESS.2019.2906751

20. R. Narayan Yadav, R. Misra and S. Bhagat. Spectrum access in cognitive smart-grid communication system with prioritized traffic, $A d$ Hoc Networks, vol. 65, pp. 38-54, Oct. 2017.

21. T. Jiang, H. Wang, M. Daneshmand and D. Wu. Cognitive Radio-Based Smart Grid Traffic Scheduling With Binary Exponential Backoff, IEEE Internet of Things Journal, vol. 4, no. 6, pp. 2038-2046, Feb. 2017.

22. M. Yigit, V. Gungor, E. Fadel, L. Nassef, N. Akkari and I. Akyildiz. Channel-aware routing and priority-aware multi-channel scheduling for WSN-based smart grid applications, Journal of Network and Computer Applications, vol. 71, pp. 50-58, Aug. 2016.

23. R. Yu, W. Zhong, S. Xie, Y. Zhang and Y. Zhang. QoS Differential Scheduling in Cognitive-Radio-Based Smart Grid Networks: An Adaptive Dynamic Programming Approach, IEEE Transactions on Neural Networks and Learning Systems, vol. 27, no. 2, pp. 435-443,Apr. 2016. 
24. S. Garlapati, T. Kuruganti, M. Buehrer and J. Reed. SMAC: A Soft MAC to Reduce Control Overhead and Latency in CDMA-Based AMI Networks, IEEE/ACM Transactions on Networking, vol. 24, no. 5, pp. 2648-2662, Oct. 2016.

https://doi.org/10.1109/TNET.2015.2481718

25. S. Biswas and V. Centeno. A Routing and Link Scheduling Strategy for Smart Grid NAN Communications, 2019 North American Power Symposium (NAPS), 2019.

26. M. Khan and M. Zeeshan. QoS-based dynamic channel selection algorithm for cognitive radio based smart grid communication network, Ad Hoc Networks, vol. 87, pp. 61-75, Mar. 2019.

https://doi.org/10.1016/j.adhoc.2018.11.007

27. M. Rafiei, S. M. Elmi and A. Zare. Wireless communication protocols for smart metering applications in power distribution networks, 2012 Proceedings of 17th Conference on Electrical Power Distribution, Tehran, 2012, pp. 1-5.

28. S. Shadravan, H. Naji and V. Bardsiri. The Sailfish Optimizer: A novel nature-inspired metaheuristic algorithm for solving constrained engineering optimization problems, Engineering Applications of Artificial Intelligence, vol. 80, pp. 20-34, Apr. 2019.

29. M. Khan, M. Zeeshan. A. Farid and M. Usman, QoS-aware traffic scheduling framework in cognitive radio based smart grids using multi-objective optimization of latency and throughput, $\mathrm{Ad} \mathrm{Hoc}$ Networks, vol. 97, p. 102020, Apr. 2020.

https://doi.org/10.1016/j.adhoc.2019.102020 